\title{
A Bayesian Approach for Energy-Based Estimation of Acoustic Aberrations in High Intensity Focused Ultrasound Treatment
}

\author{
Bamdad Hosseini $^{1, *}$, Charles Mougenot ${ }^{2}$, Samuel Pichardo ${ }^{3}$, \\ Elodie Constanciel ${ }^{4}$, James M. Drake ${ }^{4}$ and John Stockie ${ }^{5}$ \\ ${ }^{1}$ Department of Computing and Mathematical Sciences, California Institute of \\ Technology, Pasadena, CA, 91125, USA. \\ 2 Qorvo, Leidseveer 10, 3511 SB Utrecht, Netherlands. \\ 3 Radiology and Clinical Neurosciences, School of Medicine, University of Calgary, \\ AB, T2N 1N4, Canada. \\ ${ }^{4}$ Hospital for Sick Children, 555 University Avenue, Toronto, ON, M5G 1X8, Canada. \\ 5 Department of Mathematics, Simon Fraser University, Burnaby, BC, V5A 1S6, \\ Canada.
}

Received 10 January 2018; Accepted (in revised version) 16 June 2018

\begin{abstract}
High intensity focused ultrasound is a non-invasive method for treatment of diseased tissue that uses a beam of ultrasound to generate heat within a small volume. A common challenge in application of this technique is that heterogeneity of the biological medium can defocus the ultrasound beam. Here we reduce the problem of refocusing the beam to the inverse problem of estimating the acoustic aberration due to the biological tissue from acoustic radiative force imaging data. We solve this inverse problem using a Bayesian framework with a hierarchical prior and solve the inverse problem using a Metropolis-within-Gibbs algorithm. The framework is tested using both synthetic and experimental datasets. We demonstrate that our approach has the ability to estimate the aberrations using small datasets, as little as 32 sonication tests, which can lead to significant speedup in the treatment process. Furthermore, our approach is compatible with a wide range of sonication tests and can be applied to other energy-based measurement techniques.
\end{abstract}

AMS subject classifications: 65K99, 65Z05, 62G08, 62P35

Key words: Focused ultrasound, MR-ARFI, inverse problem, Bayesian, parameter estimation.

*Corresponding author. Email addresses: bamdadh@caltech.edu (B. Hosseini),

charles.mougenot@qorvo.com (C. Mougenot), samuel.pichardo@ucalgary.ca (S. Pichardo), elodie.constanciel@sickkids.ca (E. constanciel), james.drake@sickkids.ca (J. M. Drake), jstockie@sfu.ca (J. Stockie) 


\section{Introduction}

High intensity focused ultrasound (HIFU) treatment is a non-invasive method for treatment of diseased tissue. The treatment uses a focused beam of ultrasound waves that converge onto a focal point. The resulting absorption of ultrasound generates heat which in turn can ablate the targeted tissue. The method has shown clinical success in treatment of uterine fibroids [15, 20,43], prostate cancer [11], liver tumours [21, 48], brain disorders [14, 22, 28] and other medical conditions [27]. However, application of this method for treatment of brain tissue remains a challenge. Strong aberrations due to the skull bone, specifically the shift in the phase of the acoustic signal, defocus the beam and result in a loss of acoustic pressure. This problem can be resolved by estimating the introduced acoustic aberrations. If the estimate is accurate enough then one can compensate the phase of the acoustic signals (at the transducer) and refocus the beam behind the skull bone.

One approach for estimating the aberrations is to use Magnetic Resonance (MR) imaging [18] or Computed Tomography $(\mathrm{CT})[3,33$ ] to obtain a three dimensional model of the patient's skull and use this information in a computer model for acoustic wave propagation to estimate the tissue aberration and the phase shift needed to refocus the beam. However, this approach is limited by both the computational cost of the model and the accuracy of the estimates for the properties of the tissues.

An alternative approach is the so called energy-based focusing techniques of $[17,26]$. Here, Magnetic Resonance Acoustic Radiation Force Imaging (MR-ARFI) is used to obtain measurements of the intensity of the acoustic field at the focal point. MR-ARFI uses low-duty cycle HIFU pulses that generate tissue displacement in the order of microns at the focal point of the beam. The small displacement is measured with MRI using gradient pulses that encode the tissue displacement in the phase information of an MR image $[8,35]$. Using ARFI, displacement maps are generated and can be used to verify and correct the degree of focusing of HIFU beam [34]. The energy-based focusing techniques in $[17,26]$ use a dataset of displacement maps that is generated by imposing specific excitation patterns at the ultrasound transducer. Columns of a Hadamard matrix are used in [17] while [25] uses Zernink polynomials. Afterwards, the resulting displacement maps are used to estimate both the acoustic field of the transducer and the aberrations induced by the ultrasound propagation medium. The main drawback of this technique is the need for a large number of sonication tests which requires a long acquisition time for the MRI data. Recently, it was argued in [30] that energy-based techniques can be cast as a penalized least-squares problem which enables one to use more general excitation patterns. They showed that using randomized calibration sequences can reduce the number of sonication tests significantly.

In this article, a far-field approximation to the three dimensional acoustic equation is used as a forward model that can be evaluated efficiently. The effect of the tissue is modelled as an infinitely thin aberrator in front of the transducer, following [30]. These assumptions allow us to use a fast forward map that can be evaluated many times for 
the purposes of estimating the aberrations and quantifying uncertainties. Furthermore, the estimation of aberrations can be written in the form of a phase retrieval problem where the function is typically recovered from the amplitude of its Fourier transform $[16,31,32,42]$; thus, the methodology of this article is also applicable to phase retrieval problems in other applications.

The goal of this article is to demonstrate the feasibility of using a new Bayesian method to estimate the acoustic aberrations with a small number of sonication tests. The central idea is to cast the problem within the framework of Bayesian inverse problems. The Bayesian perspective provides a general framework for estimation of parameters that model the aberrations from a finite set of measurements. Appropriate models are chosen to explain the data, the measurement noise and prior knowledge of the parameters. Afterwards, an entire probability distribution on the parameters is obtained rather than a single point estimator. The Bayesian formulation can also be viewed as a generalization of the least-squares formulation of [30] (minimizers of penalized least-squares functionals are often equivalent to maximizers of the density of an underlying posterior distribution when the parameters are finite dimensional [23]). This allows for stable estimation of the aberrations with very noisy data and few sonication tests.

Furthermore, the Bayesian approach provides an estimate of the parameters as well as the associated uncertainties in that estimate. An introduction to the Bayesian perspective for solution of inverse problems and many of the techniques that are used in this article can be found in the monographs $[6,23,45]$ and the article [44] as well as the references therein. The Bayesian approach to inverse problems has been successfully applied in various areas of medical imaging such as electrical impedance tomography [24], optical diffuse tomography [2] and dynamic X-ray tomography [36] as well as other fields such as astronomy [13,39] and geoscience [12,19,45].

Our Bayesian approach consists of constructing a hierarchical smoothness prior for the aberration that reflects the prior knowledge that the aberration parameters tend to change smoothly between nearby elements on the transducer; that is, the properties of the tissue do not change dramatically between elements. Combining this prior knowledge with the forward model and the data, results in a posterior distribution which is viewed as the solution to the inverse problem. A Metropolis-within-Gibbs (MwG) sampler $[29,40]$ is used for exploring this distribution and obtaining several statistics such as the posterior mean and standard deviation of the aberrations as well as independent samples.

The remainder of this article is organized as follows. Section 2 is dedicated to the mathematical theory and the setup of the problem. A brief introduction to the far-field approximation of the acoustic equation is presented which is followed by the setup of the forward model that explains the MR-ARFI data. Next, the formulation of the Bayesian inverse problem is discussed where the likelihood and prior distributions are constructed. At the end of this section a MwG algorithm for sampling the posterior distribution is proposed. Section 3 concerns the setup of the test for synthetic and experimental conditions that were performed to verify the method. The results are then presented in Section 4 
which is followed by a discussion of the mathematical framework and the results in Section 5 .

\subsection{Notation}

Throughout this article lower case and Greek bold letters denote vectors and upper case bold letters denote matrices. Given a matrix $\mathbf{A}$ we use $\mathbf{A}^{*}$ to denote its adjoint. Finally, given $\mathbf{m} \in \mathbb{R}^{N}$ and a positive definite matrix $\boldsymbol{\Sigma} \in \mathbb{R}^{N \times N}$, we use $\mathcal{N}(\mathbf{m}, \boldsymbol{\Sigma})$ to denote a Gaussian random variable with mean $\mathbf{m}$ and covariance matrix $\Sigma$.

\section{Mathematical background and theory}

\subsection{Forward problem}

A far-field approximation to the acoustic field of a single piezoelectric ultrasound emitter is obtained under the assumption that each ultrasound emitter is infinitesimally small and emits a radially symmetric acoustic wave of amplitude $p_{0}[\mathrm{~Pa}]$ and frequency $\omega[\mathrm{Hz}]$. Then the pressure field $p(\mathbf{x})[\mathrm{Pa}]$ at a location $\mathbf{x}[\mathrm{m}]$, generated by a piezoelectric emitter at location $\mathbf{e}[\mathrm{m}]$ (see Fig. 1) is given by

$$
p(\mathbf{x} ; \mathbf{e})=z f(\mathbf{x} ; \mathbf{e}), \quad \text { where } \quad z=p_{0} \exp (i \omega t) \quad \text { and } \quad f(\mathbf{x} ; \mathbf{e})=\frac{1}{|\mathbf{x}-\mathbf{e}|} \exp \left(i \frac{\omega}{c_{0}}|\mathbf{x}-\mathbf{e}|\right) .
$$



(a)



(b)

Figure 1: (a) Schematics of the high intensity focused ultrasound setup. The transducer is submerged in an oil tank and generates an acoustic field which is directed at a phantom. The resulting displacements are measured using an MR-coil inside an MRI machine. (b) The coordinate system of the transducer that is used in Eqs. (2.1)-(2.3) for computing the acoustic field. 
Here $c_{0}[\mathrm{~m} / \mathrm{s}]$ is the speed of sound and $f(\mathbf{x} ; \mathbf{e})$ is referred to as the free-field of the emitter [30]. Interaction of the acoustic waves with the tissue results in dissipation of energy and a shift in the phase as a result of friction (micro-scale displacements) and scattering [46]. The acoustic pressure $\tilde{p}(\mathbf{x})$ in the presence of the tissue can be modelled as

$$
\tilde{p}(\mathbf{x} ; \mathbf{e})=\mu p(\mathbf{x} ; \mathbf{e}), \quad \text { where } \quad \mu=\zeta \exp (i \phi t) .
$$

Here, $\zeta$ is the amplitude coefficient related to the attenuation of the medium and $\phi$ is the shift in phase.

A physical transducer can be a phase array consisting of a large number of piezoelectric emitters. Let $N$ denote the number of physical piezoelectric emitters $(N=256$ in the case of the Philips Sonalleve V1 system (Philips, Healthcare, Vantaa, Finland) that is used in the experiments of Section 3.2) and let $\mathbf{z} \in \mathbb{C}^{N}$ denote the sonication pattern at the transducer which is the vector containing the phase and amplitude of the acoustic waves transmitted by each emitter. Also, $\mathbf{a} \in \mathbb{C}^{N}$ is defined as the vector of aberrations pertaining to each element, obtained by concatenating the $\mu$ variable of (2.2) for all elements on the transducer. Finally, define the field of view to be the displacement data of a region of $\sqrt{M} \times \sqrt{M}$ voxels around the focal point which is extracted from MR-ARFI images $(\sqrt{M}=19$ in Section 4 for the synthetic test and $\sqrt{M}=7$ for the physical experiment). Let $\tilde{\mathbf{p}} \in \mathbb{C}^{M}$ denote the measured values of the pressure at each voxel in presence of aberrations concatenated into a long vector. Then

$$
\tilde{\mathbf{p}}=\tilde{\mathbf{F}} \operatorname{diag}(\tilde{\mathbf{z}}) \mathbf{a},
$$

where $\operatorname{diag}(\tilde{\mathbf{z}})$ is the diagonal matrix created from the entries of $\tilde{\mathbf{z}}$ and $\tilde{\mathbf{F}} \in \mathbb{C}^{M \times N}$ is referred to as the free-field matrix [30] which is the mapping of the pressure in the absence of aberrations. The $i, j$ entry of $\tilde{\mathbf{F}}$ is given by the value of the free-field function $f\left(\mathbf{x}_{i} ; \mathbf{e}_{j}\right)$ in (2.1) of a piezoelectric element at location $\mathbf{e}_{i}$ evaluated at a voxel centered at $\mathbf{x}_{j}$, in the absence of the aberrator. Thus, $\tilde{\mathbf{F}}$ depends solely on the geometry of the transducer and the location of the voxels, and $\tilde{\mathbf{z}}$ are specified by the user through the design of the sonication patterns and so both $\tilde{\mathbf{F}}$ and $\tilde{\mathbf{z}}$ are known a priori.

The above model for the pressure field $\tilde{\mathbf{p}}$ relies on the assumption that tissue aberrations can be modelled as an infinitesimally thin aberrator in front of the transducer [30]. The measured displacement in MR-ARFI images is proportional to the total intensity of the signal which is equal to the square of the modulus of pressure [35]. The constant of proportionality is generally unknown but it can be estimated in a calibration step as discussed in Section 3.2.1. Throughout the remainder of this article the constant of proportionality is accounted for in the free-field matrix. To this end, let $\tilde{\mathbf{d}} \in \mathbb{R}^{M}$ be the vector of displacements at each voxel. Then

$$
\tilde{\mathbf{d}}=\operatorname{diag}(\tilde{\mathbf{p}}) \tilde{\mathbf{p}}^{*},
$$

where $\tilde{\mathbf{p}}^{*}$ denotes the element wise complex conjugate of $\tilde{\mathbf{p}}$. In practice, a finite number of $J$ sonication tests are performed where vectors $\tilde{\mathbf{z}}_{j}$ for $j=1, \cdots, J$ are prescribed as input 
at the transducer and give rise to MR-ARFI images. Each image can be summarized as a vector of displacements $\tilde{\mathbf{d}}_{j}$. These measurements constitute a dataset that is used to estimate the aberrations a. Then, a model is needed in order to relate a to the entire displacement dataset. Define the matrices

$$
\begin{aligned}
\mathbf{Z} & :=\left[\begin{array}{c}
\operatorname{diag}\left(\tilde{\mathbf{z}}_{1}\right) \\
\operatorname{diag}\left(\tilde{\mathbf{z}}_{2}\right) \\
\vdots \\
\operatorname{diag}\left(\tilde{\mathbf{z}}_{J}\right)
\end{array}\right] \in \mathbb{C}^{J N \times N,} \quad \mathbf{F}:=\mathbf{I}_{J \times J} \otimes \tilde{\mathbf{F}} \in \mathbb{C}^{J M \times J N}, \\
\mathbf{p} & :=\left[\begin{array}{c}
\tilde{\mathbf{p}}_{1} \\
\tilde{\mathbf{p}}_{2} \\
\vdots \\
\tilde{\mathbf{p}}_{J}
\end{array}\right] \in \mathbb{C}^{J M,} \quad \mathbf{d}:=\left[\begin{array}{c}
\tilde{\mathbf{d}}_{1} \\
\tilde{\mathbf{d}}_{2} \\
\vdots \\
\tilde{\mathbf{d}}_{J}
\end{array}\right] \in \mathbb{R}^{J M,}
\end{aligned}
$$

where $\mathbf{I}_{J \times J}$ is the $J \times J$ identity matrix and $\otimes$ denotes the Kronecker product. Then the forward model can be written as

$$
\mathbf{d}=\operatorname{diag}(\mathbf{p}) \mathbf{p}^{*}, \text { where } \mathbf{p}=\mathbf{F Z a} .
$$

We note that the forward model in (2.6) is an approximation to the full elastic wave equation for propagation of acoustic waves in the tissue. The accuracy and effectiveness of this approximation in the context of MR-ARFI has already been studied in detail [34]. The main benefit of this approximation is that the forward map can be evaluated efficiently which enables the use of a sampling algorithm for extraction of information in the Bayesian formulation.

\subsubsection{Relaxation to a continuous field}

Fig. 2 shows examples of phase shift and attenuation obtained with hydrophone measurements for a newborn skull using the Philips Sonalleve V1 system [9]. A schematic of the setup is depicted in Fig. 2(a) and each image (Figs. 2(b-d)) shows the measured phase shift and attenuation per transducer element mapped on a 2D projection of the transducer for a different orientation of the skull. The aberrations appear to change smoothly between the elements due to the presence of a soft spot in the skull sample. This suggests that a continuous function is a good model for the aberrations.

The acoustic elements on the emitter are arranged on a segment of a sphere (see Fig. 1). Project the location of the elements on the $x y$-plane and assume that the pair $\{\overline{\mathbf{x}}, \overline{\mathbf{y}}\}$ are vectors of the normalized $x$ and $y$ coordinates of the elements so that all of the points fit within the unit disk in $\mathbb{R}^{2}$. Furthermore, let $[-1,1]^{2}$ denote the centred unit square in $2 \mathrm{D}$ and consider a function $a:[-1,1]^{2} \rightarrow \mathbb{C}$ that is continuous. Next, define the continuous linear operator

$$
S: C\left([-1,1]^{2}\right) \rightarrow \mathbb{C}^{N}, \quad(S(a ; \overline{\mathbf{x}}, \overline{\mathbf{y}}))_{j}:=a\left(\bar{x}_{j}, \bar{y}_{j}\right),
$$




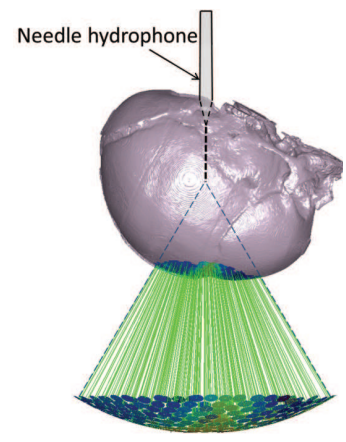

(a)
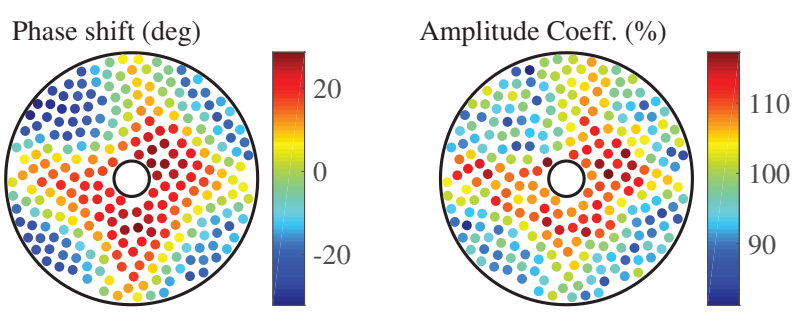

(b)

Figure 2: (a) The setup for hydrophone measurements. A hydrophone is placed inside the cavity of a skull sample and is used to capture pressure signals generated by the activation of individual transducer elements. The change in phase and amplitude are calculated by comparing these signals to measurements where the skull is removed. (b) Projection of empirical phase shift and amplitude measurements (corresponding to parameter a in (2.3)) on a newborn skull for each of the piezoelectric emitters obtained in the article [9]. The aberration changes smoothly between the elements, which motivates the assumption that the aberrations can be modelled as a continuous function. Values of amplitude higher than $100 \%$ are due to our normalization so that the average of the amplitude coefficient over the emitters is $100 \%$.

for $a \in C\left([-1,1]^{2}\right)$ and $j=1, \cdots, N$. Combining this with (2.6) defines the forward model for a continuous aberration function:

$$
\mathbf{d}=\mathcal{G}(a), \quad \mathcal{G}: C\left([-1,1]^{2}\right) \rightarrow \mathbb{R}^{J M}, \quad \mathcal{G}(a):=\operatorname{diag}(\mathbf{F Z S}(a))(\mathbf{F Z S}(a))^{*},
$$

where the dependence of $S$ on the coordinate vectors is suppressed because the entries are fixed parameters that only depend on the geometry of the device.

\subsection{Inverse problem}

We recall Bayes' rule which can be written (informally) as

$$
\pi_{\text {post }}\left(a \mid \mathbf{d}_{\mathrm{obs}}\right) \propto \pi_{\text {likelihood }}\left(\mathbf{d}_{\mathrm{obs}} \mid a\right) \pi_{\text {prior }}(a),
$$

where $\pi_{\text {prior }}$ is the prior probability distribution, reflecting prior knowledge about the parameter $a, \pi_{\text {likelihood }}$ is the likelihood distribution, indicating the probability of an observed dataset assuming that the parameter $a$ was known, and $\pi_{\text {post }}$ is the posterior distribution which is the updated distribution on $a$ given both the data and the prior. In order to avoid the delicacy of setting up the Bayesian inverse problem on the function space we only consider the discretized version of the problem where the probability distributions have well-defined Lebesgue densities and refer the reader to [44] for a detailed discussion on this subject. 


\subsubsection{Likelihood distribution $\pi_{\text {likelihood }}$}

The forward model in (2.7) does not incorporate measurement errors and therefore is not a complete model of the observed data. Assume that the error between the prediction of the forward model and the observed data, denoted by $\mathbf{d}_{\mathrm{obs}}$, can be modelled as an independent Gaussian random variable. Then

$$
\mathbf{d}_{\mathrm{obs}}=\mathcal{G}(a)+\boldsymbol{\eta}, \quad \text { where } \quad \boldsymbol{\eta} \sim \mathcal{N}(0, \boldsymbol{\Sigma}),
$$

where $\boldsymbol{\eta}$ is the measurement error and $\Sigma$ is a positive definite matrix representing the covariance of the error. The random variable $\eta$ has a density with respect to the Lebesgue measure in $\mathbb{R}^{J M}$

$$
\pi_{\boldsymbol{\eta}}(\mathbf{x})=(2 \pi)^{-\frac{I M}{2}}|\Sigma|^{-\frac{1}{2}} \exp \left(-\frac{1}{2} \mathbf{x}^{*} \Sigma^{-1} \mathbf{x}\right) .
$$

The conditional distribution of the observed data for a fixed $a$, which is simply the likelihood, is given as

$$
\pi_{\text {likelihood }}\left(\mathbf{d}_{\mathrm{obs}} \mid a\right)=\pi_{\boldsymbol{\eta}}\left(\mathbf{d}_{\mathrm{obs}}-\mathcal{G}(a)\right)=\frac{1}{\beta} \exp \left(-\Phi\left(a ; \mathbf{d}_{\mathrm{obs}}\right)\right),
$$

where

$$
\Phi\left(a ; \mathbf{d}_{\mathrm{obs}}\right):=\frac{1}{2}\left(\mathcal{G}(a)-\mathbf{d}_{\mathrm{obs}}\right)^{*} \Sigma^{-1}\left(\mathcal{G}(a)-\mathbf{d}_{\mathrm{obs}}\right),
$$

is referred to as the likelihood potential and $\beta:=(2 \pi)^{-J M / 2}|\Sigma|^{-1 / 2}$ is the normalizing constant so that $\pi_{\text {likelihood }}$ is a proper probability distribution.

\subsubsection{Hierarchical prior distribution $\pi_{\text {prior }}$}

Recall that the function $a$ was defined on the unit square $[-1,1]^{2}$. Now suppose that the box is discretized using a uniform grid of size $h$ and define the matrix $(\mathbf{A})_{j k}:=a(-1+(j-$ 1) $h,-1+(k-1) h)$ for $j, k=1,2, \cdots, \sqrt{G}$ and $\sqrt{G}=2 / h+1$, which is the usual finite difference discretization of $a$ (see Fig. 3(a)). Now, take $\mathbf{a}_{h} \in \mathbb{C}^{G}$ to be the vector that is obtained by concatenating the entries of $\mathbf{A}$ column by column and define the matrices

$$
\tilde{\mathbf{L}}:=\frac{1}{h^{2}}\left[\begin{array}{cccccc}
2 & -2 & 0 & \cdots & \cdots & 0 \\
-1 & 2 & -1 & \cdots & \cdots & 0 \\
\vdots & \vdots & & & & \vdots \\
0 & \cdots & \cdots & -1 & 2 & -1 \\
0 & \cdots & \cdots & & -2 & 2
\end{array}\right] \in \mathbb{R}^{\sqrt{G} \times \sqrt{G}} \text { and } \quad \mathbf{L}:=\tilde{\mathbf{L}} \otimes \tilde{\mathbf{L}} \in \mathbb{R}^{G \times G} \text {. }
$$

The hierarchical prior distribution is constructed by first introducing the random variables

$$
\mathbf{u} \sim \mathcal{N}\left(0, \mathbf{P}^{-1}\right), \quad \mathbf{v} \sim \mathcal{N}\left(0, \mathbf{P}^{-1}\right), \quad \alpha_{1} \sim \mathcal{N}\left(0, \sigma_{1}^{2}\right), \quad \alpha_{2} \sim \mathcal{N}\left(0, \sigma_{2}^{2}\right),
$$






(a)



(b)


Figure 3: (a) Schematic of the underlying field a discretized on a uniform grid of size $h$. The value of the field is interpolated up to each element of the transducer using a simple interpolation matrix. (b) Examples of draws from the Gaussian distribution $\mathcal{N}\left(0, \mathbf{P}^{-1}\right)$ for various values of $\gamma$ on a $50 \times 50$ grid. Smaller values of $\gamma$ result in samples with smaller features and larger amplitudes.

where $\mathbf{P}:=h\left(\mathbf{I}_{G \times G}-\gamma \mathbf{L}\right)^{2}$ and $\sigma_{1}$ and $\sigma_{2}$ are fixed. Then the prior on the aberration is represented via the random variable

$$
\mathbf{a}_{h} \sim \pi_{\text {prior }}, \quad \mathbf{a}_{h}=\mathbf{a}_{h}(\boldsymbol{\theta}):=\operatorname{diag}\left(\overline{\mathbf{u}}+\alpha_{1}^{2} \mathbf{u}\right) \exp \left(i\left(\alpha_{2}^{2} \mathbf{v}\right)\right), \quad \boldsymbol{\theta}:=\left[\begin{array}{llll}
\alpha_{1} & \alpha_{2} & \mathbf{u}^{*} & \mathbf{v}^{*}
\end{array}\right] .
$$

Here, $\overline{\mathbf{u}}$ is the prior mean of the amplification coefficient which is introduced separately since it is fixed, the exponential function is applied element by element and $\boldsymbol{\theta}$ is introduced to simplify notation in the next section.

The $\mathbf{P}^{-1}$ covariance operator in (2.14) is a finite difference discretization of the biharmonic operator $(I-\gamma \Delta)^{-2}$ with homogeneous Neumann boundary conditions. Here, $\Delta$ is the Laplacian in 2D. The finite difference matrix is scaled by a factor $h$ so that draws from the prior have the proper white noise scaling in the continuum limit as $h \rightarrow 0$. The parameter $\gamma$ controls the size of the features in the samples. Fig. 3(b) shows a few samples of $\mathcal{N}\left(0, \mathbf{P}^{-1}\right)$ for different values of $\gamma$ discretized on a $50 \times 50$ grid (that is, $G=50^{2}$ ).

We refer to $\alpha_{1}$ and $\alpha_{2}$ as hyperparameters. They control the variance of the samples, indicating prior knowledge of the range of variations of the phase shift or attenuation. Introducing the hyperparameters as multipliers is crucial to making sure that the sampling algorithm in the next section is well defined in the continuum limit [1].

It is known that in the continuum limit, draws from the Gaussian distributions in (2.14) are almost surely Lipschitz continuous [44, Lemma 6.25]. Therefore, (2.15) serves as a good model for the aberrators of Fig. 2. Since the samples are continuous, a straight forward linear interpolation can be used to obtain point values of the samples at the location of the elements. Therefore, the $S$ operator of (2.7) is easily approximated with an interpolation matrix $\mathbf{S}$. 


\subsection{Sampling the posterior distribution $\pi_{\text {post }}$}

The posterior distribution $\pi_{\text {post }}$ is, in essence, the solution to the inverse problem. However, representing this distribution is infeasible in practice since it may not have a closed form. Therefore, one often tries to extract different statistics of this distribution or simply obtain independent samples from it.

Eqs. (2.11) and (2.15) identify $\pi_{\text {post }}$ via (2.8) up to a normalizing constant. Computing this constant is often infeasible. However, Markov Chain Monte Carlo (MCMC) methods can still be used to generate samples from $\pi_{\text {post }}$ without knowledge of this normalizing constant [40]. In this article, the preconditioned Crank-Nicolson $(\mathrm{pCN})$ and MetropolisAdjusted Langevin (MALA) algorithms of [10] are used in a Metropolis within Gibbs (MwG) sampler to generate samples from $\pi_{\text {post }}$. Here, the MALA algorithm is used to sample from $\mathbf{u}$ and $\alpha_{1}$ and $\mathrm{pCN}$ is used to sample from $\mathbf{v}$ and $\alpha_{2}$. The reason for this choice is the fact that the forward map (2.7) is not differentiable with respect to the phase and so the pCN algorithm is utilized to sample in this direction. The resulting algorithm can be summarized as follows:

\section{Metropolis within Gibbs (MwG) sampler}

1. Set $k=0$ and choose $\boldsymbol{\theta}^{(0)}$ by picking $\mathbf{u}^{(0)}, \mathbf{v}^{(0)}, \alpha_{1}^{(0)}, \alpha_{2}^{(0)}$ randomly from the prior distribution and choose $\delta_{1} \in[0, \infty)$ and $\delta_{2} \in[0,1)$.

2. (MALA) Update $\mathbf{u}$ and $\alpha_{1}$ :

2.1. Propose $\boldsymbol{\theta}^{(k+1 / 4)}$ using

$$
\begin{aligned}
& \mathbf{v}^{(k+1 / 4)}=\mathbf{v}^{(k)}, \quad \mathbf{w}_{1} \sim \mathcal{N}\left(0, \mathbf{P}^{-1}\right), \\
& \mathbf{u}^{(k+1 / 4)}=\frac{2-\delta_{1}}{2+\delta_{1}} \mathbf{u}^{(k)}-\frac{2 \delta_{1}}{2+\delta_{1}} \mathbf{P}^{-1} \nabla_{\mathbf{u}} \Phi\left(\mathbf{a}_{h}^{(k)} ; \mathbf{d}_{\mathrm{obs}}\right)+\frac{\sqrt{8 \delta_{1}}}{2+\delta_{1}} \mathbf{w}_{\mathbf{1}}, \\
& \alpha_{2}^{(k+1 / 4)}=\alpha_{2}^{(k)}, \quad \xi 1 \sim \mathcal{N}\left(0, \sigma_{1}^{2}\right), \\
& \alpha_{1}^{(k+1 / 4)}=\frac{2-\delta_{1}}{2+\delta_{1}} \alpha_{1}^{(k)}-\frac{2 \delta_{1} c_{1}^{2}}{2+\delta_{1}} \nabla_{\alpha_{1}} \Phi\left(\mathbf{a}_{h}^{(k)} ; \mathbf{d}_{\mathrm{obs}}\right)+\frac{\sqrt{8 \delta_{1}}}{2+\delta_{1}} \xi_{1} .
\end{aligned}
$$

2.2. Set $\boldsymbol{\theta}^{(k+1 / 2)}=\boldsymbol{\theta}^{(k+1 / 4)}$ with probability $\kappa\left(\boldsymbol{\theta}^{(k)} ; \boldsymbol{\theta}^{(k+1 / 4)}\right)$.

2.3. Otherwise $\operatorname{set} \boldsymbol{\theta}^{(k+1 / 2)}=\boldsymbol{\theta}^{(k)}$.

3. ( $\mathrm{pCN}$ ) Update $\mathbf{v}$ and $\alpha_{2}$ : 
3.1. Propose $\boldsymbol{\theta}^{(k+3 / 4)}$ using

$$
\begin{aligned}
& \mathbf{u}^{(k+3 / 4)}=\mathbf{u}^{(k+1 / 2)}, \quad \mathbf{w}_{2} \sim \mathcal{N}\left(0, \mathbf{P}^{-1}\right), \\
& \mathbf{v}^{(k+3 / 4)}=\sqrt{1-\delta_{2}^{2}} \mathbf{v}^{(k+1 / 2)}+\mathbf{w}_{2}, \\
& \alpha_{1}^{(k+3 / 4)}=\alpha_{1}^{(k+1 / 2)}, \quad \xi_{2} \sim \mathcal{N}\left(0, \sigma_{2}^{2}\right), \\
& \alpha_{2}^{(k+3 / 4)}=\sqrt{1-\delta_{2}^{2}} \alpha_{2}^{(k+3 / 4)}+\xi_{2} .
\end{aligned}
$$

3.2. Set $\boldsymbol{\theta}^{(k+1)}=\boldsymbol{\theta}^{(k+3 / 4)}$ with probability $\tau\left(\boldsymbol{\theta}^{(k+1 / 2)} ; \boldsymbol{\theta}^{(k+3 / 4)}\right)$.

3.3. Otherwise set $\boldsymbol{\theta}^{(k+1)}=\boldsymbol{\theta}^{(k+1 / 2)}$.

4. Set $k \rightarrow k+1$ and return to step 2 .

The acceptance probability in step 2.2 is defined as

$$
\kappa(\boldsymbol{\theta} ; \tilde{\boldsymbol{\theta}}):=\min \{1, \exp (\rho(\boldsymbol{\theta} ; \tilde{\boldsymbol{\theta}})-\rho(\tilde{\boldsymbol{\theta}} ; \boldsymbol{\theta}))\},
$$

where

$$
\begin{aligned}
\rho(\boldsymbol{\theta} ; \tilde{\boldsymbol{\theta}}):=\Phi\left(\boldsymbol{\theta} ; \mathbf{d}_{\mathrm{obs}}\right)+ & \frac{1}{2}\left[\begin{array}{c}
\tilde{\alpha}_{1}-\alpha_{1} \\
h^{2}(\tilde{\mathbf{u}}-\mathbf{u})
\end{array}\right]^{*}\left[\begin{array}{c}
\nabla_{\alpha_{1}} \Phi\left(\boldsymbol{\theta} ; \mathbf{d}_{\mathrm{obs}}\right) \\
\nabla_{\mathbf{u}} \Phi\left(\boldsymbol{\theta} ; \mathbf{d}_{\mathrm{obs}}\right)
\end{array}\right] \\
& +\frac{\delta}{4}\left[\begin{array}{c}
\tilde{\alpha}_{1}+\alpha_{1} \\
h^{2}(\tilde{\mathbf{u}}+\mathbf{u})
\end{array}\right]^{*}\left[\begin{array}{c}
\nabla_{\alpha_{1}} \Phi\left(\boldsymbol{\theta} ; \mathbf{d}_{\mathrm{obs}}\right) \\
\nabla_{\mathbf{u}} \Phi\left(\boldsymbol{\theta} ; \mathbf{d}_{\mathrm{obs}}\right)
\end{array}\right] \\
& +\frac{\delta}{4}\left[\begin{array}{c}
\nabla_{\alpha_{1}} \Phi\left(\boldsymbol{\theta} ; \mathbf{d}_{\mathrm{obs}}\right) \\
\nabla_{\mathbf{u}} \Phi\left(\boldsymbol{\theta} ; \mathbf{d}_{\mathrm{obs}}\right)
\end{array}\right]^{*}\left[\begin{array}{cc}
\sigma_{1}^{2} & 0 \\
0 & \mathbf{P}^{-1}
\end{array}\right]\left[\begin{array}{c}
\nabla_{\alpha_{1}} \Phi\left(\boldsymbol{\theta} ; \mathbf{d}_{\mathrm{obs}}\right) \\
\nabla_{\mathbf{u}} \Phi\left(\boldsymbol{\theta} ; \mathbf{d}_{\mathrm{obs}}\right)
\end{array}\right] .
\end{aligned}
$$

The acceptance probability in step 3.2 is

$$
\tau(\boldsymbol{\theta} ; \tilde{\boldsymbol{\theta}}):=\min \left\{1, \exp \left(\Phi\left(\boldsymbol{\theta} ; \mathbf{d}_{\mathrm{obs}}\right)-\Phi\left(\tilde{\boldsymbol{\theta}} ; \mathbf{d}_{\mathrm{obs}}\right)\right)\right\} .
$$

Derivation of the acceptance probabilities is outside the scope of this article and the reader is referred to [10] for details.

Letting $\mathbf{a}_{h}^{(k)}:=\mathbf{a}_{h}\left(\boldsymbol{\theta}^{(k)}\right)$, the above algorithm will generate a Markov chain that has the $\pi_{\text {post }}$ as its invariant distribution [40]. This means that the samples can be used to compute the expected value of functions of the aberrations with respect to $\pi_{\text {post }}$. Suppose that the expected value of a function $f$ is of interest, then

$$
\int f(\mathbf{a}) \pi_{\text {post }}\left(\mathbf{a} \mid \mathbf{d}_{\mathrm{obs}}\right) d \mathbf{a} \approx \frac{1}{k} \sum_{\ell=1}^{k} f\left(\mathbf{a}_{h}^{(\ell)}\right) .
$$


The functions of interest for practical applications are the posterior mean $\mathbf{a}_{\mathrm{PM}}$, covariance $\operatorname{Cov}(\mathbf{a})$ and standard deviation $\operatorname{std}(\mathbf{a})$ of the aberration for each element:

$$
\begin{aligned}
& \mathbf{a}_{\mathrm{PM}} \approx \frac{1}{k} \sum_{\ell=1}^{k} \mathbf{S a}_{h}^{(\ell)}, \\
& \operatorname{Cov}(\mathbf{a}) \approx \frac{1}{k} \sum_{\ell=1}^{k}\left(\mathbf{S a}_{h}^{(\ell)}-\mathbf{a}_{P M}\right)\left(\mathbf{S a}_{h}^{(\ell)}-\mathbf{a}_{P M}\right)^{*}, \\
& \operatorname{std}(\mathbf{a})_{i} \approx\left(\operatorname{diag}(\operatorname{Cov}(\mathbf{a}))_{i}\right)^{1 / 2} \quad \text { for } \quad i=1,2, \cdots, N .
\end{aligned}
$$

Recall that $\mathbf{S}$ is the discrete approximation of the pointwise evaluation operator $S$ in (2.7) as discussed in Section 2.2.2.

A key detail in implementation of the $\mathrm{MwG}$ algorithm is computing the derivative of the likelihood potential in step 2.1. This gradient can be calculated by solving an adjoint problem. Let $\mathbf{G}:=\mathbf{F Z S} \operatorname{diag}\left(\exp \left(i \alpha_{2}^{2} \mathbf{v}\right)\right)$ and recall that the pressure field can be written as

$$
\mathbf{p}=\mathbf{F Z S} \operatorname{diag}\left(\overline{\mathbf{u}}+\alpha_{1}^{2} \mathbf{u}\right) \exp \left(i\left(\alpha_{2}^{2} \mathbf{v}\right)\right) .
$$

Then, straightforward calculations show that

$$
\nabla_{\alpha_{1}} \mathcal{G}(\boldsymbol{\theta})=2 \alpha_{1} \operatorname{diag}(\mathbf{p}) \mathbf{G}^{*} \mathbf{u}, \quad \nabla_{\mathbf{u}} \mathcal{G}(\boldsymbol{\theta})=2 \alpha_{1}^{2} \operatorname{Re}\left[\operatorname{diag}(\mathbf{p}) \mathbf{G}^{*}\right] .
$$

Combining this with (2.12) gives

$$
\left[\begin{array}{c}
\nabla_{\alpha_{1}} \Phi\left(\boldsymbol{\theta} ; \mathbf{d}_{\mathrm{obs}}\right) \\
\nabla_{\mathbf{u}} \Phi\left(\boldsymbol{\theta} ; \mathbf{d}_{\mathrm{obs}}\right)
\end{array}\right]=\left[\begin{array}{c}
\nabla_{\alpha_{1}} \mathcal{G}(\boldsymbol{\theta})^{*} \\
\nabla_{\mathbf{u}} \mathcal{G}(\boldsymbol{\theta})^{*}
\end{array}\right] \boldsymbol{\Sigma}^{-1}\left(\mathcal{G}(\boldsymbol{\theta})-\mathbf{d}_{\mathrm{obs}}\right) .
$$

Therefore, every step of the MALA update costs roughly twice as much as the pCN update but MALA is more efficient in exploring the posterior.

\section{Methods}

In this section we describe the details of two experiments that were performed to test our framework for estimation of the acoustic aberrations. The first test uses a synthetic dataset of the displacement map which is generated by the same model as the forward model of Section 2.1. In the second test we use a physical dataset that was obtained using a Philips Sonalleve V1 device.

\subsection{Test with synthetic displacement map}

The first test was performed using a synthetic dataset generated with the target aberrator in Fig. 2(b). The goal here was to test the algorithm in a more relaxed setting where there was no discrepancy between the forward model and the model for the data. 
Generating the synthetic dataset involves many details including the geometry of the transducer and the location of the focal point that are besides the main point of this article. To keep the discussion short, we only present a summary of the methodology for performing the synthetic experiments. The first step in generating the artificial dataset was to identify the free-field matrix of the transducer $\tilde{\mathbf{F}}$ using (2.1) and the location of the elements and the MR-ARFI voxels; The $k$-th column of $\tilde{\mathbf{F}}$ is simply the free-field corresponding to the contribution of the $k$-th ultrasound emitter evaluated at the center of each voxel in the MR-ARFI images. Afterwards, the matrix $\mathbf{F}$ is constructed using (2.5). The next step was to construct the design matrix $\mathbf{Z}$ which is identified by the $\tilde{\mathbf{z}}_{j}$ vectors. This matrix contains the prescribed values of the amplitude and phase of the acoustic waves at the transducer. The virtual elements of [17] were used to group nearby piezoelectric emitters and construct the design matrix. Let $\mathbf{H}_{256}$ denote the $256 \times 256$ Hadamard matrix [17, Eq. 8] with columns $\mathbf{h}_{1}$ to $\mathbf{h}_{256}$. Then $\tilde{\mathbf{z}}_{j}=\mathbf{h}_{1}+\exp \left(i \frac{\pi}{6}\right) \mathbf{h}_{j}$ for $j=2, \cdots, 16$.

A noisy version of the design vectors was considered with

$$
\tilde{\mathbf{z}}_{j}^{\text {noisy }}:=\tilde{\mathbf{z}}_{j}+\boldsymbol{\epsilon}_{1} \exp \left(i \frac{\pi}{4} \boldsymbol{\epsilon}_{2}\right), \text { where } \boldsymbol{\epsilon}_{1}, \boldsymbol{\epsilon}_{2} \sim \mathcal{N}\left(0,(0.05)^{2} \cdot \mathbf{I}_{256 \times 256}\right) .
$$

This added an extra layer of noise that is not accounted for in the formulation of the inverse problem. The standard deviation of the noise in the phase was taken to be 0.05 as a reasonable estimate of the errors in imposing the sonication patterns in practical settings. Putting these vectors together gave a noisy design matrix $\mathbf{Z}^{\text {noisy }}$ and the synthetic dataset was generated using

$$
\mathbf{d}_{\text {obs }}^{\text {art }}=\mathbf{F Z}^{\text {noisy }} \mathbf{a}^{\text {art }}+\boldsymbol{\eta}, \quad \text { where } \quad \boldsymbol{\eta} \sim \sigma_{\text {obs }}^{\text {art }} \cdot \mathcal{N}\left(0,(0.2)^{2} \cdot \mathbf{I}_{256 \times 256}\right),
$$

and $\sigma_{\mathrm{obs}}^{\text {art }}$ is the standard deviation of $\mathbf{F Z}^{\text {noisy }} \mathbf{a}^{\text {art }}$. The standard deviation of the measurement noise was taken to be 0.2 which implied a signal to noise ratio of 5 . This choice was made to replicate data that is highly noisy. Fig. 4 shows examples of the generated noisy data along with the prescribed phase and amplitudes of the elements.

A total of 16 sonication tests were performed in this example, that is, the design matrix had 16 columns. In order to solve the inverse problem, a coarse $8 \times 8$ mesh $(h=1 / 4)$ was used to discretize the aberration field $a$. It was assumed that the noise covariance $\boldsymbol{\Sigma}=\left(0.15 \cdot \sigma_{\mathrm{obs}}^{\text {art }}\right)^{2} \cdot \mathbf{I}_{J M \times J M}$. The prior mean was taken to be $\overline{\mathbf{u}}=1$, the prior wave number $\gamma=1 / 5$ and the hyperparameter prior variances were $\sigma_{1}=\sigma_{2}=2$. The step sizes in the MCMC algorithm were $\delta_{1}=4 \times 10^{-5}$ and $\delta_{2}=5 \times 10^{-3}$ resulting in an average acceptance probability of 0.32 across the two Metropolis Hastings updates.

\subsection{Test with MR-ARFI displacement map}

A test was done using the displacement maps obtained from MR-ARFI data which was acquired using a Philips Sonalleve V1 ultrasound system (Philips Healthcare, Vantaa, Finland) and an Achieva 3T MRI machine (Philips, Best, Netherlands). A phased array transducer consisting of 256 elements was used with a focal length of $12 \mathrm{~cm}$ and aperture 

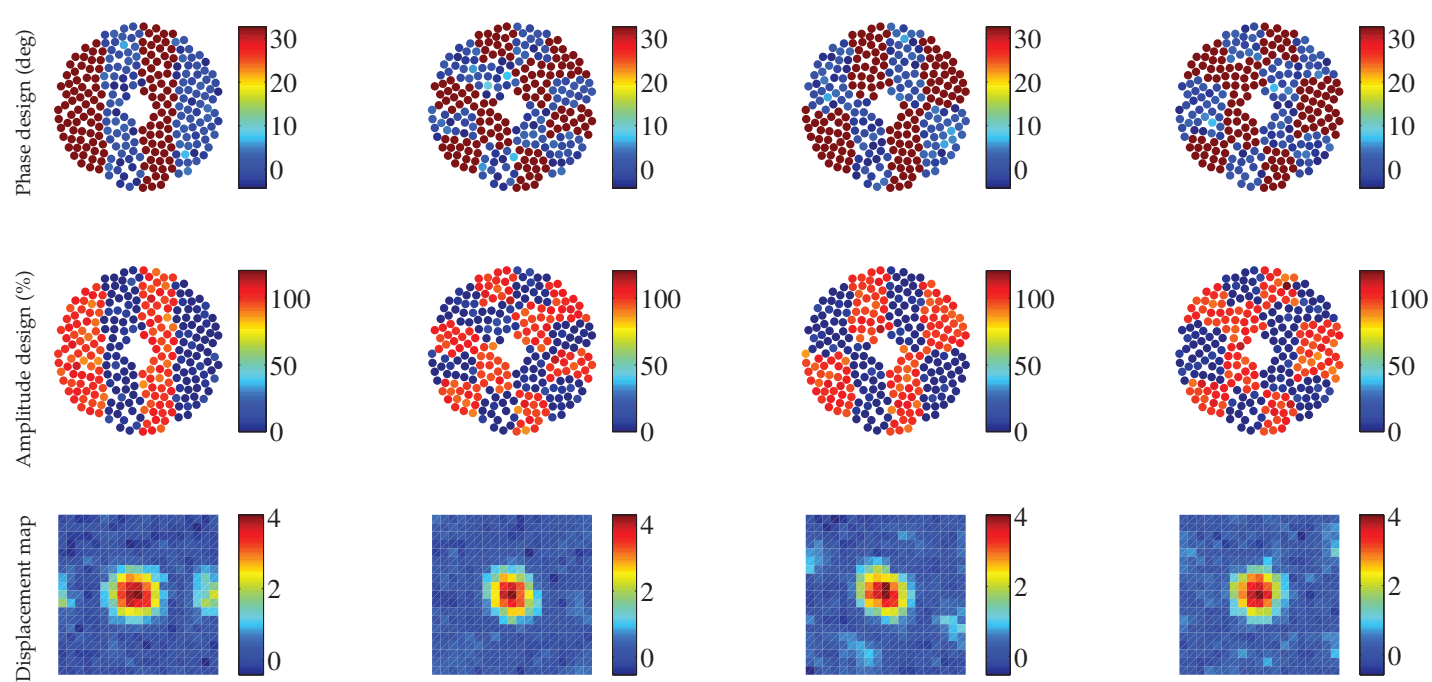

Figure 4: A few examples of artificial experiments, indicating the prescribed noisy phase and amplitude (the $\tilde{\mathbf{z}}_{j}^{\text {noisy }}$ vectors) along with the displacement map (the resulting MR image) of the focal point (subsets of $\mathbf{d}_{\text {obs }}^{\text {art }}$ ) scaled by the standard deviation of the images. The displacement maps show the focal point before refocusing.

of $13 \mathrm{~cm}$. The transducer was operating at $1.2 \mathrm{MHz}$ generating ultrasound pulses of $1 \mathrm{~ms}$ ranging from 0 to 300 acoustic Watts. The transducer was submerged in an oil tank (as illustrated in Fig. 1) and the acoustic field was targeted at a $4 \mathrm{~cm}$ phantom which mimics muscle tissue (CIRS, Norfolk, VA, USA). The displacement due to the ultrasound pulses was measured using a dedicated MR receiver coil of $4 \mathrm{~cm}$ inner diameter. The displacements were measured using MR-ARFI sequences based on a RF-spoiled gradient-recalled Echo-Planar Imaging (EPI) sequence with Repetition Time (TR) of $42 \mathrm{~ms}$, Echo Time (TE) of $30 \mathrm{~ms}$, flip angle of 20 degrees, EPI factor of 9, MRI resolution of $64 \times 64$ pixels, bi-polar motion encoding gradients of $1 \mathrm{~ms}$ in duration and amplitude of $30 \mathrm{mT} / \mathrm{m}$. Images were obtained every 0.26 seconds from a single slice that was orthogonal to the path of the beam with a field of view of $180 \times 180 \mathrm{~mm}^{2}$ and voxels of size $0.7 \times 0.7 \times 4 \mathrm{~mm}^{3}$.

At first, a set of measurements was taken without an aberrator. This dataset is used for estimating the free-field matrix of the transducer and so it is referred to as the calibration dataset. To obtain this dataset the phase stepping technique of $[17,26]$ was used with 16 virtual elements (the first 16 columns of the Hadamard matrix) with ten steps in phase for each virtual element. Thus, this dataset consists of 160 sonication patterns. A cosine function was then fit to the displacement of each voxel during the phase stepping in order to retrieve the phase and estimate the free-field matrix of each element.

Afterwards, a second set of measurements was taken in the presence of the aberrator of Fig. 2(a) consisting of 32 sonication patterns. This dataset is referred to as the reconstruction dataset. The input phase and amplitude of the 256 elements were modified to imitate the effect of the target aberrator instead of using a physical aberrator in front of 
the beam. That is, the aberrator was added to the prescribed excitation patterns during each sonication test. This approach is advantageous because the values of the aberration parameters are known and so we can easily assess the quality of the estimates. Programming of the HIFU system and collection of MR images were performed using the toolboxes MatHIFU and MatMRI [49]. Here, the design matrix $\mathbf{Z}$ was constructed from the vectors $\tilde{\mathbf{z}}_{j}=\frac{1}{2}\left(\mathbf{h}_{1}+\mathbf{h}_{j}\right)$ for $j=1, \cdots, 16$ and $\left.\tilde{\mathbf{z}}_{j}=\frac{1}{2}\left(\mathbf{h}_{1}+\exp \left(i \frac{\pi}{3}\right)\right) \mathbf{h}_{j}\right)$ for $j=17, \cdots, 32$. All tests were performed with a field of view of $7 \times 7$ voxels, corresponding to $\sqrt{M}=7$. Only a small portion of the images are used from each frame since signal to noise ratio drops rapidly for voxels that are far from the focal point. Each measurement was repeated ten times and then averaged in order to reduce the noise.

A consequence of the phase stepping method is that displacement maps are normalized and so the dataset does not include any information regarding the amplitude coefficients. To this end, the inverse problem is only solved for the phase shift and hyperparameter $\alpha_{2}$. The noise covariance in the formulation of the inverse problem was taken to be $\boldsymbol{\Sigma}=\left(0.2 \cdot \sigma_{\mathrm{obs}}\right)^{2} \cdot \mathbf{I}_{J M \times J M}$ where $\sigma_{\mathrm{obs}}$ is the standard deviation of the reconstruction dataset. Recall that here, $M=49$ (number of voxels) and $J=16$ (number of images). As before, an $8 \times 8$ grid was used for discretization of the aberration field $a$. The prior mean was taken to be $\overline{\mathbf{u}}=1$ and the prior wave number $\gamma=1 / 5$. The prior variances on the hyperparameters were $\sigma_{1}=0.5$ and $\sigma_{2}=0.5$ and the MCMC step sizes were $\delta_{1}=1.1 \times 10^{-5}$ and $\delta_{2}=1.5 \times 10^{-3}$ resulting in an average acceptance probability of 0.54 across the two Metropolis Hastings updates. Since inversion is performed solely for the phase shift, only the $\mathrm{pCN}$ update was utilized.

\subsubsection{Calibration of the free-field matrix}

The free-field matrix of the transducer was computed using the phase stepping technique of $[17,26]$ but the estimated field is often not accurate enough to give a satisfactory estimate of the aberrations. This issue is amplified when measurements are noisy and the number of sonication tests is significantly smaller than the number of elements on the transducer. Furthermore, the MR-ARFI data consists of measurements of displacement while the forward model of Section 2.1 is valid for acoustic intensity. Although acoustic intensity is expected to be proportional to displacement [35], the constant of proportionality is unknown.

These discrepancies will manifest as an apparent aberrator in front of the beam. For example, running the algorithm on the calibration dataset would still estimate a significant value for the aberrations. In practice this aberrator must be estimated in a calibration step before computing the actual aberrator using the reconstruction dataset. This will also automatically estimate the constant of proportionality between intensity and displacement. Here, the posterior mean of this inherent aberrator, denoted by $\mathbf{a}_{\text {calibration, }}$ is computed over the first 32 measurements of the calibration dataset. Once this vector is available, the calibration can be performed by simply replacing the matrix $\mathbf{S}$ by

$$
\mathbf{S}_{\text {calibrated }}=\operatorname{diag}\left(\mathbf{a}_{\text {calibration }}\right) \mathbf{S} \text {. }
$$




\subsection{Assessing the quality of refocusing}

Take the posterior mean $\mathbf{a}_{\mathrm{PM}}$ to be a good estimator of the true aberration $\mathbf{a}$, and let $\phi_{\mathrm{PM}}$ and $\phi$ be their corresponding phase shift vectors. Define the vectors

$$
\begin{aligned}
& \mathbf{e}_{1}:=\operatorname{diag}\left[\tilde{\mathbf{F}} \exp \left(i\left(\boldsymbol{\phi}-\boldsymbol{\phi}_{\mathrm{PM}}\right)\right)\right] \tilde{\mathbf{F}} \exp \left(i\left(\boldsymbol{\phi}-\boldsymbol{\phi}_{\mathrm{PM}}\right)\right), \\
& \mathbf{e}_{2}:=\operatorname{diag}[\tilde{\mathbf{F}} \exp (i \boldsymbol{\phi})] \tilde{\mathbf{F}} \exp (i \boldsymbol{\phi}), \\
& \mathbf{e}_{3}:=\operatorname{diag}[\tilde{\mathbf{F}} \mathbf{1}] \tilde{\mathbf{F}} \mathbf{1},
\end{aligned}
$$

and the expected improvement $E I$ and expected recovery $E R$ functionals for the posterior mean

$$
E I\left(\mathbf{a}_{\mathrm{PM}}\right):=\left(1-\frac{\left\|\mathbf{e}_{3}\right\|_{\infty}-\left\|\mathbf{e}_{1}\right\|_{\infty}}{\left\|\mathbf{e}_{3}\right\|_{\infty}-\left\|\mathbf{e}_{2}\right\|_{\infty}}\right) \times 100, \quad E R\left(\mathbf{a}_{\mathrm{PM}}\right):=\left(\frac{\left\|\mathbf{e}_{1}\right\|_{\infty}-\left\|\mathbf{e}_{2}\right\|_{\infty}}{\left\|\mathbf{e}_{3}\right\|_{\infty}}\right) \times 100 .
$$

EI measures the percentage of lost intensity that is recovered while ER measures how the maximum intensity of the refocused beam compares to the the maximum intensity of a perfectly focused beam. Since power is directly related to beam intensity, the expected improvement $E I$ can be viewed as a measure of improvement in treatment efficiency. Furthermore, based on Pennes bio-heat law [38], beam intensity is proportional to the temperature increase at the focal point. This temperature increase has an exponential relationship to the tissue damage based on thermal dose model [41]. Then the expected recovery $E R$ can be viewed as a measure of improvement in required treatment dosage after refocusing. These measures are used in the Section 4 to further assess the performance of the estimated aberrations.

\section{Results}

\subsection{Test with synthetic displacement map}

A summary of Bayesian posterior statistics using the synthetic dataset is presented in Fig. 5. Posterior mean and standard deviations are computed using $5 \times 10^{5}$ samples with a burn-in period of $3 \times 10^{3}$ samples (that is, the first $3 \times 10^{3}$ samples were discarded since the Markov chain had not yet converged at that point). The posterior mean (Fig. 5(b)) is taken to be a good estimator of the actual value of the parameters. This is supported by Fig. 5(c) which is the pointwise absolute difference between the posterior mean and the target aberrator. Here the maximum error in the phase is 21 degrees while the average error (among the elements) is 4.5 degrees. As for the attenuation, the maximum error is 45 percent and the average error is 14 percent. Compare these values to Fig. 5(d) which depicts the standard deviation of the aberration parameters and can be taken as a measure of uncertainty in the approximations. The standard deviation of the aberrations is close to the average point wise absolute error. Therefore, the standard deviation is a good measure of the accuracy of $\mathbf{a}_{\mathrm{PM}}$. Furthermore, Fig. 5(f) compares the prediction of 


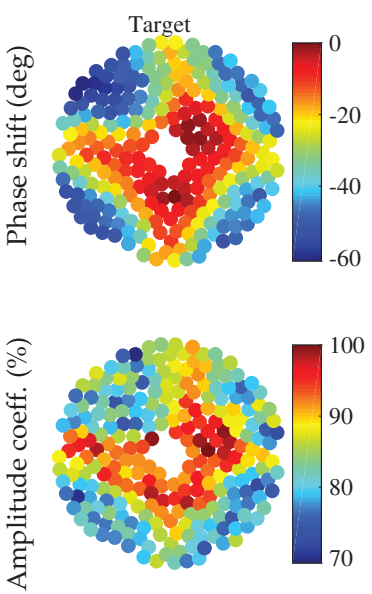

(a)
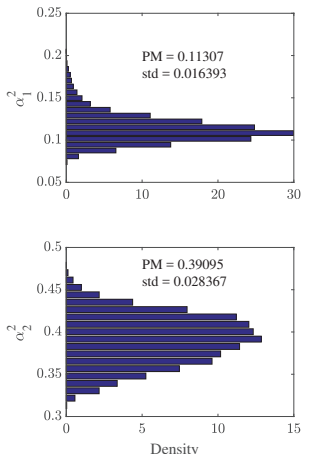

(e)
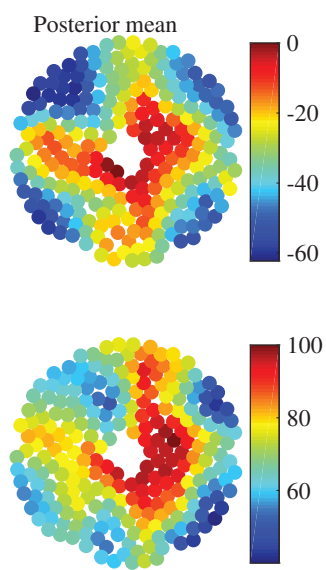

(b)
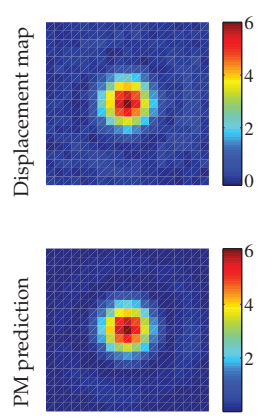

(f)
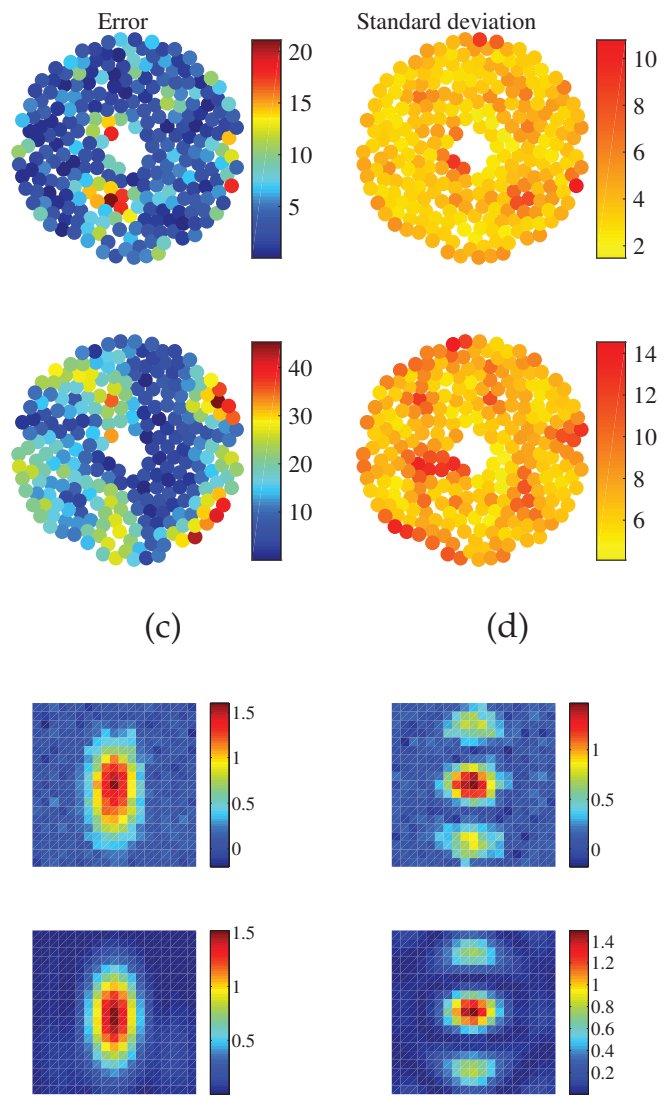

(c)

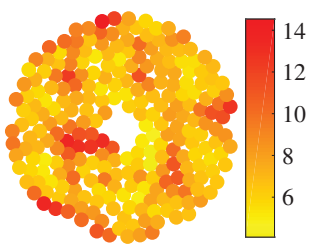

(d)
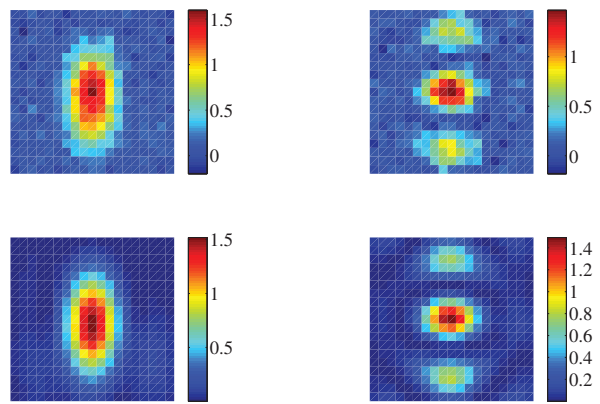

Figure 5: A summary of Bayesian posterior statistics using the synthetic dataset. (a) The target aberrator used for generating the data. (b) Posterior mean (PM) of the aberration parameters along with (c) the pointwise difference between the posterior mean and the target aberrator. (d) The standard deviation (std) of the posterior samples which is indicative of the level of uncertainty. (e) Estimated marginal posterior distributions on the hyperparameters. (f) A few examples of the synthetic displacement map at the focal point compared with the prediction of the posterior mean of the aberrator.

the data using a $\mathbf{a}_{\mathrm{PM}}$ to the actual data set on a few frames of the MR-ARFI data. This shows a good agreement between the prediction and the data and further certifies the choice of apM as a pointwise estimator of the target. Finally, the expected improvement $E I\left(\mathbf{a}_{\mathrm{PM}}\right) \approx 71 \%$ and $E R\left(\mathbf{a}_{\mathrm{PM}}\right) \approx 5 \%$. Therefore, using $\mathbf{a}_{\mathrm{PM}}$ to refocus the beam recovers $71 \%$ of the lost intensity. However, this only improves maximum intensity by $5 \%$ which is due to the fact that the aberrator of Fig. 5(a) is very weak and the defocused beam is already at $93 \%$ intensity.

In the case of the hyperparameters, one can integrate out the rest of the parameters and directly estimate the marginal posterior distribution as in Fig. 5(e). Here we demon- 

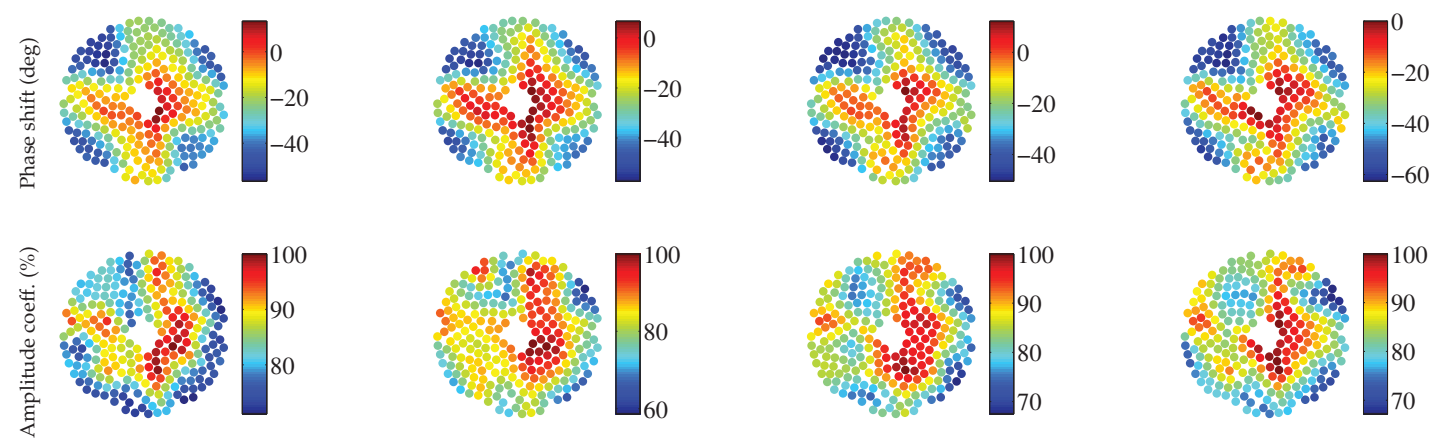

Figure 6: A few samples from $\pi_{\text {post }}$ on the aberration parameters. The samples are $10^{5}$ steps apart in the Markov chain so that they can be treated as independent.

strate the marginals on $\alpha_{1}^{2}$ and $\alpha_{2}^{2}$ rather than $\alpha_{1}$ and $\alpha_{2}$ as these are the standard deviations of the fields $\mathbf{u}$ and $\mathbf{v}$ and are more physically relevant. These results indicate a drop of two orders of magnitude in the standard deviation of the hyperparameters, compared with the prior standard deviation, indicating that the value of the hyperparameters are computed with high confidence.

In addition to the above statistics, one can also look at independent samples from $\pi_{\text {post }}$ as depicted in Fig. 6 . These samples are generated by choosing individual samples from the Markov chain that are far enough apart. The distance between the samples in Fig. 6 was chosen large enough so that the integrated autocorrelation function of the chain was below $10^{-3}$. In this case the distance was taken to be $10^{5}$ steps based on the worst integrated autocorrelation function in Fig. 10(a). The independent samples can be taken as examples of aberrators that are likely to have generated the dataset and provide further insight regarding $\pi_{\text {post }}$. It is clear that the samples have very similar features in comparison to the mean. For example, there are no discontinuities or multi-modal behavior. This further supports the choice of the posterior mean apM as a point estimator for the true value of the parameters.

\subsection{Test with MR-ARFI displacement map}

Figures 8 to 10 show a similar summary of the results for the test with MR-ARFI displacement map as was shown for the test with synthetic displacement maps. Here the results were computed using $5 \times 10^{5}$ samples from the Markov chain with a burn-in period of $3 \times 10^{5}$. Fig. $7(\mathrm{~b})$ shows the posterior mean of the phase shift $\phi_{\mathrm{PM}}$ which is once again taken to be a good estimator of the true phase shift. Fig. 7(c) depicts the pointwise absolute error between $\phi_{\mathrm{PM}}$ and the target. Here, the maximum error in the phase is 45 degrees and the average error across the elements is 19 degrees. The errors here are notably larger as compared to the test with the synthetic displacement map, especially in the case of the attenuation. This is most likely due to large discrepancies between the 


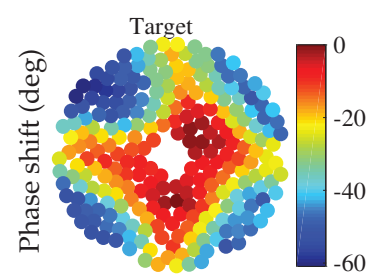

(a)

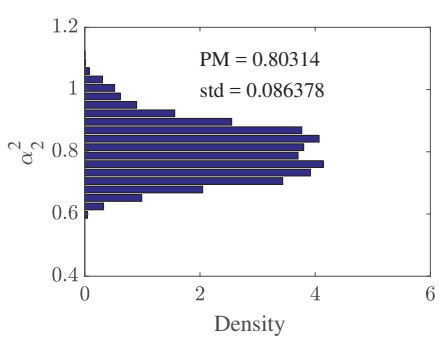

(e)

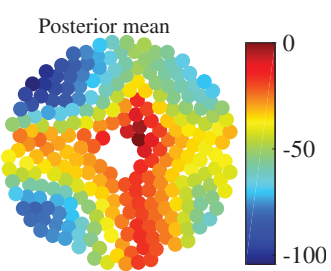

(b)

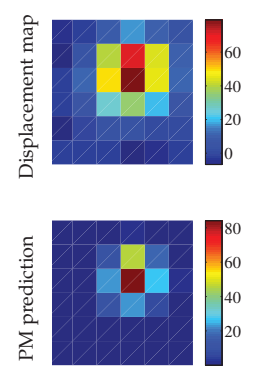

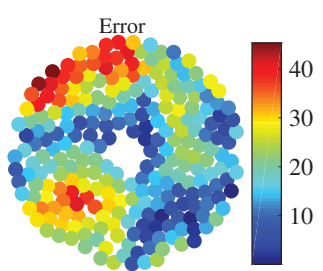

(c)
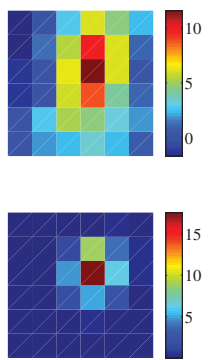

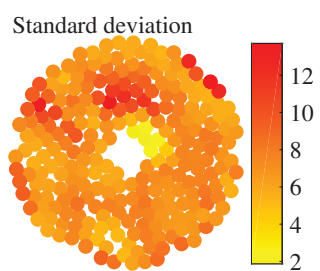

(d)
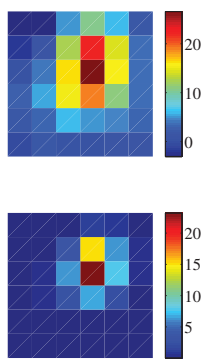

(f)

Figure 7: A summary of Bayesian posterior statistics using the experimental MR-ARFI dataset. (a) The target aberrator imposed on the transducer. (b) The posterior mean (PM) phase shift and (c) pointwise difference between the posterior mean and the target aberrator. (d) The standard deviation of the posterior. (e) The marginal distribution of the hyperparameters estimated using the Markov chain. (f) A comparison between a few frames of the MR-ARFI displacement map and the prediction of the forward model at the posterior mean.

forward model and the measured MR-ARFI data. Nevertheless, the overall shape of the aberrator and range of phase shifts are captured.

Fig. 7(d) shows an estimate of the standard deviation of the aberrations under $\pi_{\text {post }}$ indicating a possible error of plus or minus 12 degrees. It should be noted that this error is still smaller than the true pointwise error and the algorithm underestimate the true uncertainty of the solution. Fig. 7(e) shows the posterior marginal on the hyperparameters. As before, the posterior standard deviation on the hyperparameters has been reduced significantly as compared to that of the prior distribution which is a sign that these parameters are well identified by the data. To check whether the prediction of $\mathbf{a}_{\mathrm{PM}}$ matches the data, a few frames of the MR-ARFI images are compared to the prediction at the posterior mean in Fig. 7(f). The matching between the data and the prediction was adequate at the focal point but deteriorates away from it. Specifically, the prediction obtained the correct range of variations of each frame and the right location for the point of maximum intensity.

For this dataset $E I\left(\mathrm{a}_{\mathrm{PM}}\right) \approx 42 \%$ which predicts a good recovery of the intensity. However, expected recovery $E R\left(\mathbf{a}_{\mathrm{PM}}\right) \approx 3 \%$ which is smaller in comparison to the synthetic test above. This is expected since the estimate of the aberrator is not as accurate as before due to discrepancies between the model and the physical data. 

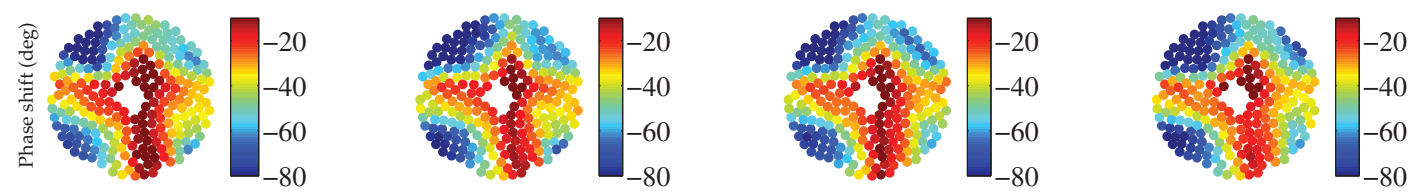

Figure 8: Four samples from the Markov chain on phase shift after the burn-in period with the experimental MR-ARFI dataset. The samples are $10^{5}$ steps apart so that they can be treated as independent.

Finally, a few independent samples from $\pi_{\text {post }}$ are presented in Fig. 8. The samples are $10^{5}$ steps apart in order to ensure their independence. This choice was based on the slowest decaying integrated autocorrelation function in Fig. 10(b). Once again, comparing the samples to the posterior mean indicates that the samples are close to the posterior mean and so $\mathbf{a}_{\mathrm{PM}}$ is a reasonable point estimator for the parameter values.

\section{Discussion}

\subsection{Mathematical framework}

The most notable feature of the forward problem in (2.6) is that $\mathbf{d}$ is a non-linear function of the aberration a. This is an attribute of the MR-ARFI data which makes the inverse problem more challenging to solve. In particular, this means that one-shot methods for computing the minimizers of regularized least squares functionals (such as the common Tikhonov regularization) are no longer applicable. In this case one can use numerical optimization algorithms such as Newton's method or the L-BFGS algorithm $[37,47]$ to find a minimizer but this choice requires a modification of the formulation to get around the non-differentiability of the forward map with respect to the phase.

Another effective method for estimating the phase shift, which lies within the optimization category, is the matrix completion approach of [7]. Matrix completion recasts the phase retrieval problem as a penalized least squares problem in a high dimensional space with a linear forward map (2.6). The resulting solution is a low-rank square matrix and the phase shift is given by the first eigenvector of that matrix. In Fig. 9 we present a comparison between the Bayesian approach and the matrix completion method for the synthetic dataset of Section 3.1, where the matrix completion problem was solved using the TFOCS package $[4,5]$. We note first that matrix completion is more efficient than the Bayesian approach, since the minimizer can be found in about 15 minutes while the Bayesian method requires an hour to obtain an stable estimate of the posterior mean and variance (both computations were performed on an Apple MacBook Pro laptop with a 2.3 GHz dual-core Intel Core i5 processor). However, the Bayesian solution appears to be significantly more accurate. Furthermore, a comparison to Fig. 5(d) shows that the uncertainty estimate obtained using the Bayesian method serves as a certificate that indicates the order of the error in the Bayesian estimate; such an uncertainty estimate is not available for matrix completion. Finally, we tuned the regularization parameter in the 

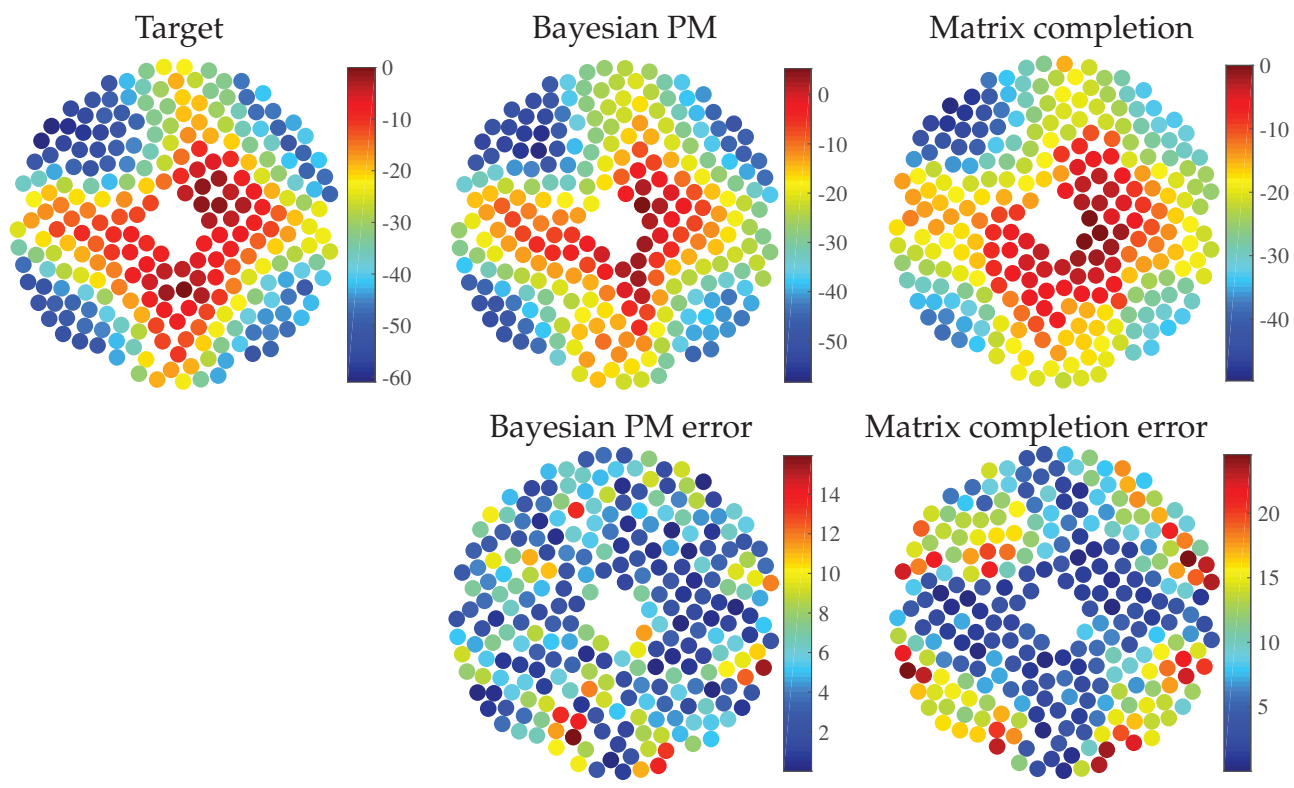

Figure 9: Comparison between phase shift estimators obtained using the Bayesian approach and the matrix completion method of [7] (top row), along with the corresponding absolute error between the target and estimate (bottom row). All values are reported in degrees.

matrix completion problem to obtain a good estimate of the phase shift. The Bayesian method on the other hand estimates the hyperparameters automatically.

From a practical perspective, our formulation of the forward and inverse problems offer complete freedom in the choice of the number of sonications tests $J$, the size of the MRI images $M$, the size of the parameter space for the aberrations $G$ and the number of elements $N$. It is often preferable to take $J$ to be as small as possible to reduce the time required to collect the MRI data. It is also desirable to choose $G$ to be small in order to reduce the computational cost of the algorithms. Taking $M$ to be large means that each MRI image will have more information. However, the signal to noise ratio drops rapidly for voxels that are far from the focal point. This is the reason why the dataset in the MRARFI experiments uses a smaller field of view compared to the artificial example. Then the choice of each one of these parameters requires further study in future.

\subsection{Performance of the MCMC algorithm}

The $\mathrm{pCN}$ algorithm is a modification of the random walk Metropolis Hastings algorithm that is well defined on a function space [10]. It tends to generate samples that are highly correlated and so it explores the posterior distribution slowly. However, the pCN update does not require derivative information and therefore can be used to sample from nondifferentiable densities. This also means that each step of $\mathrm{pCN}$ is relatively inexpensive. The MALA algorithm utilizes an optimal proposal step that results in less correlated 

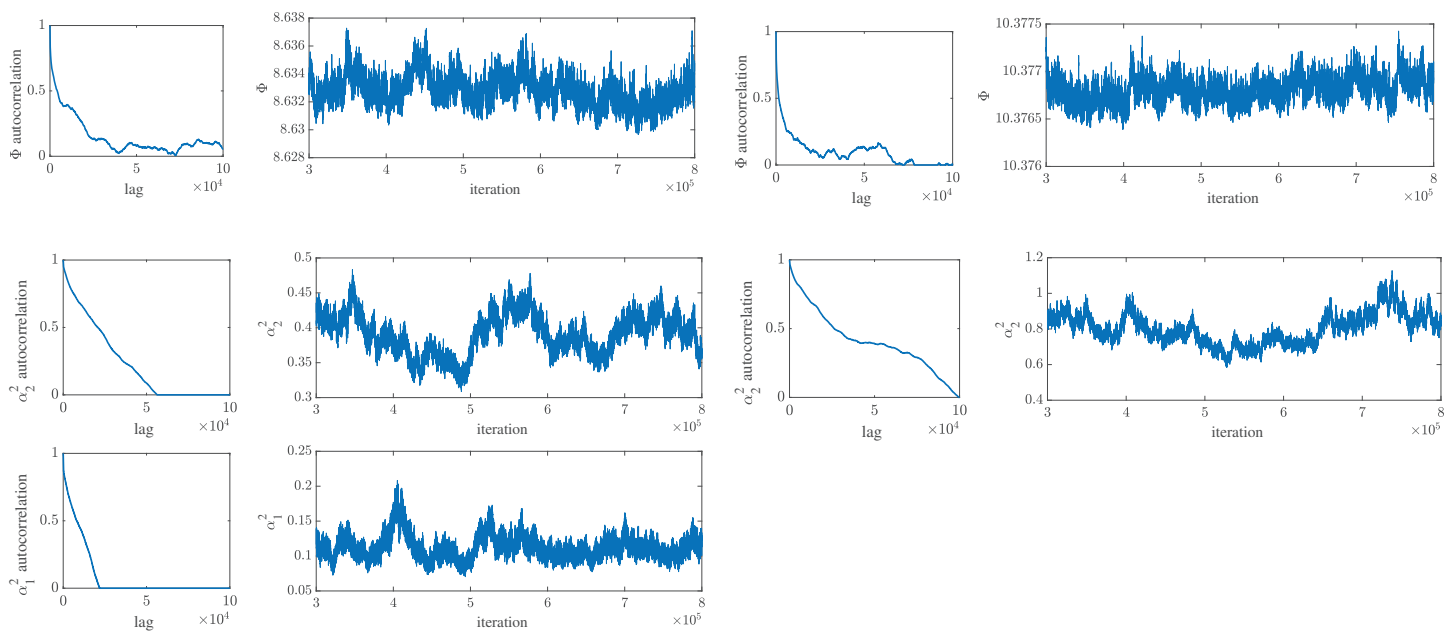

(a)

(b)

Figure 10: Autocorrelation and trace plots of the likelihood potential and hyperparameters using (a) the synthetic dataset and (b) the experimental MR-ARFI displacement map.

samples [10,40] which makes the algorithm better at exploring the posterior distribution $\pi_{\text {post }}$ but each step of the algorithm has a higher computational cost. This cost becomes significant when a large dataset is at hand, because this increases the cost of gradient computations.

The difference between the two steps of the algorithm is apparent in Fig. 10(a) where the integrated autocorrelation functions and trace plots are presented for the likelihood potential $\Phi$ and the squared hyperparameters $\alpha_{1}^{2}$ and $\alpha_{2}^{2}$ in the test with the synthetic dataset. The integrated autocorrelation of $\alpha_{2}^{2}$ decays slower than that of $\alpha_{1}^{2}$. This is likely because samples from the latter are generated using the MALA updates. The $\alpha_{2}^{2}$ chain is slow because in this case the data is fairly informative in the direction of phase shift, meaning that the posterior distribution $\pi_{\text {post }}$ is dominated by the likelihood rather than the prior distribution. Looking at the trace plots in Fig. 10, it is interesting to note that the $\Phi$ chain demonstrates better mixing in comparison to the chains for $\alpha_{1}^{2}$ and $\alpha_{2}^{2}$. We observe a similar behavior in the case of the physical dataset.

The results of this article were obtained using an implementation of the MwG algorithm for estimation of the aberrator in MATLAB on a personal laptop (MacBook Pro with a $3 \mathrm{GHz}$ Intel Core i7 processor and $8 \mathrm{~GB}$ or memory) in less than two hours. This processing time may not be ideal for practical settings but it can be effectively reduced by implementation of the algorithm in more efficient languages and applying techniques such as parallel tempering and population MCMC [29] to further reduce the required sample size needed to compute the expectations. 


\subsection{Quality of the estimates with synthetic and physical datasets}

In Section 3.1 the results with a synthetic dataset were presented. This example is viewed as an idealized setting where there is little discrepancy between the process for generation of the data and the forward model. The reconstructions were adequate even in the presence of large errors, the posterior mean apM of the phase (Fig. 5(b)) was close to the actual value of the aberrations (the average pointwise error of the phase estimate was 4.5 degrees amounting to $15 \%$ relative error). Furthermore, the estimated standard deviation appeared to be a good estimate of the expected errors in the reconstruction. However, in the case of the attenuation, the posterior mean $\mathbf{a}_{\mathrm{PM}}$ was not as accurate as the phase shift even in this idealized setting (compare Fig. 5(a) and (b)). This was due to the fact that the displacement data was not sensitive to relatively small changes in the attenuation.

In Section 4 we used the expected improvement $E I$ and expected recovery $E R$ functionals as a measure of the quality of reconstructions. Recall that $E I$ is a measure of improvement in power efficiency while $E R$ is a measure of improvement in required dosage. In the synthetic and physical tests we recovered 3 to $5 \%$ of the total intensity after refocusing. This was partly due to the fact that we used a weak aberrator that reduced the beam intensity by $7 \%$, so that the beam was already at $93 \%$ intensity without any focusing. Of course this makes our aberrator harder to estimate as compared to a stronger aberrator. However, even in this setting, a 3 to $5 \%$ improvement in beam intensity results in a similar gain in power efficiency but has a more significant impact in dosage efficiency due to the fact that tissue damage grows exponentially [41] as intensity (and in turn temperature) increases at the focal point.

Finally, we note that the computed free-field matrix of the transducer and the calibration step have a significant impact on the quality of the reconstructions. In the test with experimental MR-ARFI displacement map of Section 3.2 the free-field was estimated using 160 sonication tests with ten steps in phase for each virtual elements. Using subsets of this data resulted in a less accurate free-field matrix which in turn resulted in very different reconstructions. Then one might prefer to use a large dataset in order to obtain an accurate estimate of the free-field matrix which can be used on a smaller reconstruction dataset later on. The fact that calibration can be done offline makes this a viable approach in practical settings.

\subsection{Future research}

The framework presented in this article can be extended in multiple directions in order to improve the quality of the reconstructions which, in turn, will lead to better focusing of the beam. Obtaining an accurate estimate of the empirical free-field with the minimum number of sonication tests is a crucial task. The estimates of the aberrations become more sensitive to the free-field matrix as the dataset becomes smaller. Therefore, a good estimate of the free-field matrix is needed in order to further reduce the number sonication tests that are performed under in vivo or clinic conditions. 
Another promising direction for future research is improving the sampling algorithm. MCMC algorithms often have a difficult time traversing high dimensional distributions. In such cases, strategies such as population or adaptive MCMC or parallel tempering [29] can be used to improve the statistical performance of the chain. Alternatively, one can also improve the algorithm by changing the forward model so that it is differentiable in the phase. This would allow the use of the MALA update on the entire posterior which would greatly improve the performance.

On the topic of MR-ARFI experiments, one can explore multiple directions for improving the quality of the dataset. An interesting question is the interplay between voxel size, measurement noise and acquisition duration. Smaller voxels give a better estimate of the free-field matrix and the aberration but they are associated with more noise. This, in turn, requires smaller phase steps and longer acquisition time or perhaps more averaging steps per sonication test. Therefore, finding the optimal parameters for generating the dataset remains a challenge in practice.

\section{Acknowledgments}

The authors would like to thank Profs. Nilima Nigam and Chris Budd for fruitful discussions. BH and CM are thankful to the Fields Institute and the organizers of the FieldsMprime Industrial Problem Solving Workshop during the August of 2014, where their collaboration was initiated. Finally, this work was supported in part by the Natural Sciences and Engineering Research Council of Canada, the Brain Canada Multi-Investigator Research Initiative and the Focused Ultrasound Foundation.

\section{References}

[1] S. Agapiou, J. M. Bardsley, O. Papaspiliopoulos, and A. M. Stuart. Analysis of the Gibbs sampler for hierarchical inverse problems. SIAM/ASA Journal on Uncertainty Quantification, 2(1):511-544, 2014.

[2] S. R. Arridge, J. P. Kaipio, V. Kolehmainen, M. Schweiger, E. Somersalo, T. Tarvainen, and M. Vauhkonen. Approximation errors and model reduction with an application in optical diffusion tomography. Inverse Problems, 22:175-195, 2006.

[3] J. F. Aubry, M. Tanter, M. Pernot, J. L. Thomas, and M. Fink. Experimental demonstration of noninvasive trans-skull adaptive focusing based on prior computed tomography scans. Journal of the Acoustical Society of America, 113(1):84-93, 2003.

[4] S. R. Becker, E. J. Candès, and M. C. Grant. Templates for convex cone problems with applications to sparse signal recovery. Mathematical Programming Computation, 3(3):165, 2011.

[5] S. R. Becker, E. J. Candès, and M. C. Grant. TFOCS: Templates for first-order conic solvers. http://cvxr.com/tfocs/, May 2018.

[6] D. Calvetti and E. Somersalo. An Introduction to Bayesian Scientific Computing: Ten Lectures on Subjective Computing, volume 2. Springer Science and Business Media, New York, 2007.

[7] E. J. Candès, Y. C. Eldar, T. Strohmer, and V. Voroninski. Phase retrieval via matrix completion. SIAM Review, 57(2):225-251, 2015. 
[8] J. Chen, R. Watkins, and K. B. Pauly. Optimization of encoding gradients for MR-ARFI. Magnetic Resonance in Medicine, 63(4):1050-1058, 2010.

[9] E. Constanciel Colas, A. C. Waspe, C. Mougenot, T. Looi, S. Pichardo, and J. M. Drake. Mapping of insertion losses and time-of-flight delays of pediatric skulls using a clinical MRguided high intensity focused ultrasound system. In International Society for Therapeutic Ultrasound, Utrecht, Netherlands, April 2015.

[10] S. L. Cotter, G. O. Roberts, A. M. Stuart, and D. White. MCMC methods for functions: modifying old algorithms to make them faster. Statistical Science, 28(3):424-446, 2013.

[11] S. Crouzet, F. J. Murat, G. Pasticier, P. Cassier, J. Y. Chapelon, and A. Gelet. High intensity focused ultrasound (HIFU) for prostate cancer: current clinical status, outcomes and future perspectives. International Journal of Hyperthermia, 26(8):796-803, 2010.

[12] T. Cui, C. Fox, and M. J. O'Sullivan. Bayesian calibration of a large-scale geothermal reservoir model by a new adaptive delayed acceptance Metropolis Hastings algorithm. Water Resources Research, 47(10):W10521, 2011.

[13] M. C. Edwards, R. Meyer, and N. Christensen. Bayesian parameter estimation of core collapse supernovae using gravitational wave simulations. Inverse Problems, 30(11):114008, 2014.

[14] W. J. Elias, D. Huss, T. Voss, J. Loomba, M. Khaled, E. Zadicario, R. C. Frysinger, S. A. Sperling, S. Wylie, S. J. Monteith, et al. A pilot study of focused ultrasound thalamotomy for essential tremor. New England Journal of Medicine, 369(7):640-648, 2013.

[15] F. M. Fennessy and C. M. Tempany. A review of magnetic resonance imaging-guided focused ultrasound surgery of uterine fibroids. Topics in Magnetic Resonance Imaging, 17(3):173$179,2006$.

[16] J. R. Fienup. Phase retrieval algorithms: a personal tour. Applied Optics, 52(1):45-56, 2013.

[17] E. Herbert, M. Pernot, G. Montaldo, M. Fink, and M. Tanter. Energy-based adaptive focusing of waves: application to noninvasive aberration correction of ultrasonic wavefields. IEEE Transactions on Ultrasonics, Ferroelectrics and Frequency Control, 56(11):2388-2399, 2009.

[18] K. Hynynen and J. Sun. Trans-skull ultrasound therapy: The feasibility of using imagederived skull thickness information to correct the phase distortion. IEEE Transactions on Ultrasonics, Ferroelectrics, and Frequency Control, 46(3):752-755, 1999.

[19] M. A. Iglesias, K. Lin, and A. M. Stuart. Well-posed Bayesian geometric inverse problems arising in subsurface flow. Inverse Problems, 30(11):114001, 2014.

[20] M. E. Ikink, M. J. Voogt, H. M. Verkooijen, P. N. M. Lohle, K. J. Schweitzer, A. Franx, P. T. M. Willem, L. W. Bartels, and M. A. A. J. van den Bosch. Mid-term clinical efficacy of a volumetric magnetic resonance-guided high-intensity focused ultrasound technique for treatment of symptomatic uterine fibroids. European Radiology, 23(11):3054-3061, 2013.

[21] R. O. Illing, J. E. Kennedy, F. Wu, G. R. Ter Haar, A. S. Protheroe, P. J. Friend, F. V. Gleeson, D. W. Cranston, R. R. Phillips, and M. R. Middleton. The safety and feasibility of extracorporeal high-intensity focused ultrasound (HIFU) for the treatment of liver and kidney tumours in a western population. British Journal of Cancer, 93(8):890-895, 2005.

[22] D. Jeanmonod, B. Werner, A. Morel, L. Michels, E. Zadicario, G. Schiff, and E. Martin. Transcranial magnetic resonance imaging-guided focused ultrasound: noninvasive central lateral thalamotomy for chronic neuropathic pain. Neurosurgical Focus, 32(1):E1, 2012.

[23] J. Kaipio and E. Somersalo. Statistical and Computational Inverse Problems. Springer Science and Business Media, New York, 2005.

[24] J. P. Kaipio, V. Kolehmainen, E. Somersalo, and M. Vauhkonen. Statistical inversion and Monte Carlo sampling methods in electrical impedance tomography. Inverse Problems, 
16(5):1487-1522, 2000.

[25] E. A. Kaye, Y. Hertzberg, M. Marx, B. Werner, G. Navon, M. Levoy, and K. B. Pauly. Application of Zernike polynomials towards accelerated adaptive focusing of transcranial high intensity focused ultrasound. Medical Physics, 39(10):6254-6263, 2012.

[26] B. Larrat, M. Pernot, G. Montaldo, M. Fink, and M. Tanter. MR-guided adaptive focusing of ultrasound. IEEE Transactions on Ultrasonics, Ferroelectrics, and Frequency Control, 57(8):17341747, 2010.

[27] B. Liberman, D. Gianfelice, Y. Inbar, A. Beck, T. Rabin, N. Shabshin, G. Chander, S. Hengst, R. Pfeffer, A. Chechick, et al. Pain palliation in patients with bone metastases using MRguided focused ultrasound surgery: a multicenter study. Annals of Surgical Oncology, 16(1):140-146, 2009.

[28] N. Lipsman, M. L. Schwartz, Y. Huang, L. Lee, T. Sankar, M. Chapman, K. Hynynen, and A. M. Lozano. MR-guided focused ultrasound thalamotomy for essential tremor: a proofof-concept study. The Lancet Neurology, 12(5):462-468, 2013.

[29] J. S. Liu. Monte Carlo Strategies in Scientific Computing. Springer Series in Statistics. Springer, New York, 2008.

[30] N. Liu, A. Liutkus, J.-F. Aubry, L. Marsac, M. Tanter, and L. Daudet. Random calibration for accelerating MR-ARFI guided ultrasonic focusing in transcranial therapy. Physics in Medicine and Biology, 60(3):1069, 2015.

[31] S. Marchesini. Invited article: A unified evaluation of iterative projection algorithms for phase retrieval. Review of Scientific Instruments, 78(1):011301, 2007.

[32] S. Marchesini. Phase retrieval and saddle-point optimization. Journal of the Optical Society of America A, 24(10):3289-3296, 2007.

[33] F. Marquet, M. Pernot, J. F. Aubry, G. Montaldo, L. Marsac, M. Tanter, and M. Fink. Noninvasive transcranial ultrasound therapy based on a 3D CT scan: protocol validation and in vitro results. Physics in Medicine and Biology, 54(9):2597-2614, 2009.

[34] L. Marsac, D. Chauvet, B. Larrat, M. Pernot, B. Robert, M. Fink, A.-L. Boch, J.-F. Aubry, and M. Tanter. MR-guided adaptive focusing of therapeutic ultrasound beams in the human head. Medical Physics, 39(2):1141-1149, 2012.

[35] N. McDannold and S. E. Maier. Magnetic resonance acoustic radiation force imaging. Medical Physics, 35(8):3748-3758, 2008.

[36] E. Niemi, M. Lassas, and S. Siltanen. Dynamic X-ray tomography with multiple sources. In 8th International Symposium on Image and Signal Processing and Analysis (ISPA), pages 618-621. IEEE, 2013.

[37] J. Nocedal and S. J. Wright. Numerical Optimization. Springer, New York, 2nd edition, 2006.

[38] H. H. Pennes. Analysis of tissue and arterial blood temperatures in the resting human forearm. Journal of applied physiology, 1(2):93-122, 1948.

[39] S. Pursiainen and M. Kaasalainen. Sparse source travel-time tomography of a laboratory target: accuracy and robustness of anomaly detection. Inverse Problems, 30(11):114016, 2014.

[40] C. Robert and G. Casella. Monte Carlo Statistical Methods. Springer Science and Business Media, New York, 2013.

[41] S. A. Sapareto and W. C. Dewey. Thermal dose determination in cancer therapy. International Journal of Radiation Oncology Biology Physics, 10(6):787-800, 1984.

[42] Y. Shechtman, Y. C. Eldar, O. Cohen, H. N. Chapman, J. Miao, and M. Segev. Phase retrieval with application to optical imaging: a contemporary overview. IEEE Signal Processing Magazine, 32(3):87-109, 2015.

[43] E. A. Stewart, J. Rabinovici, C. M. C. Tempany, Y. Inbar, L. Regan, B. Gastout, G. Hesley, H. S. 
Kim, S. Hengst, and W. M. Gedroye. Clinical outcomes of focused ultrasound surgery for the treatment of uterine fibroids. Fertility and Sterility, 85(1):22-29, 2006.

[44] A. M. Stuart. Inverse problems: a Bayesian perspective. Acta Numerica, 19:451-559, 2010.

[45] A. Tarantola. Inverse Problem Theory and Methods for Model Parameter Estimation. SIAM, Philadelphia, 2005.

[46] G. ter Haar and C. Coussios. High intensity focused ultrasound: physical principles and devices. International Journal of Hyperthermia, 23(2):89-104, 2007.

[47] C. R. Vogel. Computational Methods for Inverse Problems. SIAM, Philadelphia, 2002.

[48] F. Wu, Z.-B. Wang, W.-Z. Chen, W. Wang, Y. Gui, M. Zhang, G. Zheng, Y. Zhou, G. Xu, M. Li, et al. Extracorporeal high intensity focused ultrasound ablation in the treatment of 1038 patients with solid carcinomas in China: an overview. Ultrasonics Sonochemistry, 11(3):149154, 2004.

[49] B. Zaporzan, A. C. Waspe, T. Looi, C. Mougenot, A. Partanen, and S. Pichardo. MatMRI and MatHIFU: software toolboxes for real-time monitoring and control of MR-guided HIFU. Journal of Therapeutic Ultrasound, 1(1):1-12, 2013. 